(C) 2006 IEEE. Personal use of this material is permitted. Permission from IEEE must be obtained for all other uses, in any current or future media, including reprinting/republishing this material for advertising or promotional purposes, creating new collective works, for resale or redistribution to servers or lists, or reuse of any copyrighted component of this work in other works. 


\title{
Design of Switching Damping Controllers for Power Systems Based on a Markov Jump Parameter System Approach
}

\author{
R. A. Ramos, L. Li, V. A. Ugrinovskii, and H. R. Pota
}

\begin{abstract}
The application of a new technique, based on the theory of Markov Jump Parameter Systems (MJPS), to the problem of designing controllers to damp power system oscillations is presented in this paper. This problem is very difficult to address, mainly because these controllers are required to have an output feedback decentralized structure. The technique relies on the statistical knowledge about the system operating conditions to provide less conservative controllers than other modern robust control approaches. The influence of the system interconnections over its modes of oscillation is reduced by means of a proper control design formulation involving Integral Quadratic Constraints. The discrete nature of some typical events in power systems (such as line tripping or load switching) is adequately modeled by the MJPS approach, therefore allowing the controller to withstand such abrupt changes in the operating conditions of the system, as shown in the results.
\end{abstract}

\section{INTRODUCTION}

The problem of low frequency electromechanical oscillations in power systems has been challenging engineers and researchers for several decades. Such oscillations became increasingly common after the 1960 decade, when the interconnection of previously isolated systems became usual, aiming at better operation reliability and optimization of generation resources.

Nowadays, with deregulation processes leading to stronger competition in the power industry and great pressures to meet environmental requirements, the degrading effect of these oscillations for the power system operation tends to get even worse. The reason for that relates to the nonlinear effects observed in such operation which, when pushed closer to its limits, often produces results quite different from those predicted by linear models. However, in spite of that, linear models are still used to represent the power system behavior, both in analysis and controller design studies, largely due to their simplicity (when compared to the more accurate nonlinear models).

A typical practice of power industries is to assess this oscillation problem using the design of supplementary stabilizing controllers to enhance the system natural damping under

This work was supported by the Australian Research Council and the University of New South Wales at the Australian Defence Force Academy (UNSW@ADFA), being carried out during the appointment of Dr. R. A. Ramos as a Visiting Fellow at the UNSW@ADFA.

R. A. Ramos is with Escola de Engenharia de São Carlos - USP, São Carlos, SP, 13566-590, Brazil ramosesel.eesc.usp.br

L. Li, V. A. Ugrinovskii and H. R. Pota are with the School of Information Technology and Electrical Engineering, the University of New South Wales at the Australian Defence Force Academy, Canberra, ACT 2600, Australia l.li@adfa.edu.au, valu@adfa.edu.au, h-potaladfa.edu.au these stressed conditions, therefore preventing it from going unstable as an effect of these oscillations. The commonly used controllers are called Power System Stabilizers [1], which are designed by means of classical control techniques involving phase compensation (therefore relying on linear time invariant models).

The need for better assessment of this problem, mainly with respect to the robustness of the designed controllers (so they can withstand significant variations in the system operating conditions, as well as those caused by system nonlinearities, while still maintaining an acceptable performance), has been well recognized among the scientific community over the past years [2]. Nevertheless, this is a very difficult control problem to address, mainly due to its several practical requirements [3]. Among the most important requirements is the output-feedback decentralized structure of the controllers. This requirement is unavoidable because some of the generator states are referred to a common reference and cannot be reliably measured and transmitted over the large distances that separate power plants in typical systems. Many different proposals of new methodologies, capable of providing such robust controllers, have been reported in the literature ([4] and [5] are examples).

Most of the proposals based on modern robust control approaches are intended as general solutions for the wide variety of situations that can happen during the operation of the system. They generally assume a poor knowledge about the operating point and try to derive controllers capable of stabilizing all possible operating conditions accounted for in the uncertain model. Very often this uncertain model contains descriptions of operating points that would never be observed in practice. It is conceivable, then, that such methodologies would tend to generate very conservative controllers. Indeed, this is observed in the vast majority of methodologies based on modern robust control approaches. Overly conservative controllers are not welcome to the field, for they implicate in excessively large control efforts, usually not achievable due to the existing limitations in controller outputs.

A recently developed technique [6], [7] based on the minimax theory of Markov Jump Parameter Systems (MJPS) provides a way to overcome this conservativeness, and this is the key concept presented in this paper. By recognizing that some problems in power system operation (which could lead to potentially dangerous oscillations) have an inherently hybrid system nature and by accounting for it in the controller design, it is possible to generate controllers that while being less conservative than other robust control approaches, can still provide an acceptable performance for 
the power system operation. Moreover, having in mind that interconnections play an important role in the oscillation problem, the presented approach also tries to reduce their negative impact on the system damping by means of a formulation involving Integral Quadratic Constraints (IQCs) [9].

This paper is organized as follows: Section II depicts the power system modeling aspects relevant to the work reported here, Section III presents the theoretical fundamentals and the algorithm of the proposed technique and Section IV provides a successful application example of this technique. The paper finishes with some concluding remarks in Section V.

\section{Power System Model}

Power system operation can be modeled at several different levels of complexity, depending on the intended application for the model. In this work, a multimachine model (typically employed in electromechanical oscillation studies) was used, for it is generally accepted that this model can adequately represent the most significant dynamic behaviors involved in the problem.

Traditionally, in small-signal stability assessment, generators can be described by the one-axis model, and a constant impedance model is used to represent the loads [10]. Both practices were also employed in this work. Thyristor excited Automatic Voltage Regulators (AVRs), without transient gain reduction [11], were also used to compose the model. The transmission network was considered as a passive circuit, and therefore it was modeled using algebraic constraints, representing interconnections between dynamic models of the generators; nonlinear state-space descriptions were used to represent the latter. Dynamics of the i-th generator in this model can be described by the following set of equations:

$$
\begin{aligned}
\dot{\delta}_{i}= & \omega_{0} \omega_{i}-\omega_{0} \\
\dot{\omega}_{i}= & \frac{1}{2 H_{i}}\left[P_{m i}-E_{q i}^{\prime}\left(I_{R i} \cos \delta_{i}+I_{I i} \sin \delta_{i}\right)-D_{i} \omega_{i}\right] \\
\dot{E}_{q i}^{\prime}= & \frac{1}{\tau_{d o i}^{\prime}}\left[K_{a i}\left(V_{o i}-V_{r e f i}+V_{s i}\right)-E_{q i}^{\prime}+\right. \\
& \left.\left(x_{d i}-x_{d i}^{\prime}\right)\left(I_{I i} \cos \delta_{i}-I_{R i} \sin \delta_{i}\right)\right] \\
\dot{V}_{o i}= & \frac{1}{T_{r i}}\left[V_{o i}-\left(E_{q i}^{\prime 2}+2 E_{q i}^{\prime} x_{d i}^{\prime}\left(I_{I i} \cos \delta_{i}-I_{R i} \sin \delta_{i}\right)+\right.\right. \\
& \left.\left.x_{d i}^{\prime 2}\left(I_{R i}^{2}+I_{I i}^{2}\right)\right)^{1 / 2}\right]
\end{aligned}
$$

In (1)-(4), $\delta_{i}$ is the rotor angle, $\omega_{i}$ is the rotor speed (with respect to a synchronous reference), $E_{q i}^{\prime}$ is the quadratureaxis transient voltage, $V_{o i}$ is the terminal voltage transducer output and $V_{s i}$ is the stabilizing signal for the AVR. Definitions of the parameters in these equations can be found in [10]. Since mechanical powers are modeled as constant inputs, one of the machines is considered as an infinite bus, providing both an angular reference and the necessary corrections of power imbalances for the system. $I_{R i}$ and $I_{I i}$ are, respectively, the real and imaginary parts of the stator current, with respect to the angular reference of the infinite bus, and are given by

$$
\begin{aligned}
I_{R i} & =\sum_{k=1}^{n}\left(G_{i k} E_{q k}^{\prime} \cos \delta_{k}-B_{i k} E_{q k}^{\prime} \sin \delta_{k}\right) \\
I_{I i} & =\sum_{k=1}^{n}\left(B_{i k} E_{q k}^{\prime} \cos \delta_{k}+G_{i k} E_{q k}^{\prime} \sin \delta_{k}\right)
\end{aligned}
$$

where $n$ is the total number of generators in the system and the definitions of $G_{i k}$ and $B_{i k}$ can also be found in [10]. Complex nonlinearities can be observed when (5)-(6) are substituted into (1)-(4). Single and double summations appear in the resulting equations as a consequence of the substitution of algebraic constraints (representing the transmission network) into the dynamic equations. Therefore, it is obvious that interconnections are strongly related to the nonlinear behavior of power systems.

Reducing the detrimental impact of interconnections over the natural damping of the generators is a key to achieving a good system performance. In the present work, this goal will be achieved by imposing upper bounds on the allowable effects of these interconnections, expressed in the mathematical form of IQCs.

Furthermore, as mentioned in Section I, the technique applied in this paper uses some knowledge of the system operating conditions to reduce the conservatism of the designed controllers. More specifically, the fact that power system operation is best described by a continuous-time model subject to discrete events is explicitly taken into account by using a MJPS approach for the system model.

Scheduled maintenance or unexpected outage of transmission lines are examples of situations in which discrete events occur. For both cases, the industry has very good statistical data available, describing the frequency and approximate probability of occurrence of such events. This knowledge can be used to produce an adequate MJPS description for the power system, consisting of linearized models for each mode of operation, with their associated transition probability rates.

For the technique applied in this paper, such MJPS description is composed by $N$ subsystems $S_{i}$, each related to a particular generator of the system, in a particular operating condition. In the following, generators will be referred to as subsystems and operating conditions as modes. The linearized models forming the MJPS description of the system are as follows:

$$
S_{i}:\left\{\begin{array}{l}
\dot{x}_{i}=A_{i}(\eta(t)) x_{i}(t)+B_{i}(\eta(t)) u_{i}(t)+ \\
E_{i}(\eta(t)) \xi_{i}(t)+L_{i}(\eta(t)) r_{i}(t), \\
z_{i}=C_{i}(\eta(t)) x_{i}(t)+D_{i}(\eta(t)) u_{i}(t), \\
\zeta_{i}=H_{i}(\eta(t)) x_{i}(t)+G_{i}(\eta(t)) u_{i}(t), \\
y_{i}=C_{y, i}(\eta(t)) x_{i}(t)+D_{y, i}(\eta(t)) \xi_{i}(t) .
\end{array}\right.
$$

In (7), $x_{i} \in \mathbf{R}^{n_{i}}$ is the state, $u_{i} \in \mathbf{R}^{m_{i}}$ is the control input, $\xi_{i} \in \mathbf{R}^{p_{i}}$ is the perturbation (which could describe any local uncertainty within its respective subsystem), $\zeta_{i} \in \mathbf{R}^{h_{i}}$ is the uncertainty output, $z_{i} \in \mathbf{R}^{q_{i}}$ is the controlled output, $y_{i} \in \mathbf{R}^{p_{i}}$ is the measured output, the input $r_{i}$ describes the effect of 
subsystems $S_{j}, j \neq i$, on the subsystem $S_{i}$, and $\eta(t)$ describes the mechanism of mode switching in the system.

For the application reported in this paper, the states $x_{i}$ are given by $x_{i}=\left[\Delta \delta_{i} \Delta \omega_{i} \Delta E_{q i}^{\prime} \Delta V_{o i}\right]^{\prime}$, the control input $u_{i}$ is the stabilizing signal $\Delta V_{s i}$ and the measured output $y_{i}$ is the rotor speed $\Delta \omega_{i}$, where $\Delta$ denotes deviation from the respective equilibrium value. The controlled output is composed of both the system states and control inputs with appropriate weighting matrices. No local uncertainties in the state equations and no uncertainty outputs were considered in the particular application reported in Section IV, although there is provision in the developed procedure for taking them into account. In the remainder of this section and in the following section, the technique is presented in a general case which accounts for the presence of local uncertainties.

It is assumed that $\eta(t)$ is a homogeneous stationary Markov chain defined in a complete probability space $(\Omega, \mathscr{F}, \mathscr{P})$ and taking values in a finite set $\mathbb{K}=\{1,2, \cdots, k\}$. Its state transition probability matrix $\mathbf{P}(t)=[\mathscr{P}\{\eta(t+s)=$ $\mu \mid \eta(s)=v\}]_{v, \mu=1}^{k}$ is independent of $s \geq 0$ and under mild conditions is known to have the form $\mathbf{P}(t)=e^{\mathbf{Q} t}$, where $\mathbf{Q}:=\left[q_{v \mu}\right]_{v, \mu=1}^{k}$ is a matrix in which $q_{v \mu} \geq 0, v \neq \mu$ and $q_{v v}=-\Sigma_{\mu \neq v} q_{v \mu}$. The stationary initial distribution $\pi=$ $\left[\pi_{1}, \cdots, \pi_{k}\right]$ of the process $\eta(t)$ will be assumed to be positive, i.e., $\pi_{j}>0, \forall j \in \mathbb{K}$. The matrix $Q$ and vector $\pi$ reflect the statistics of mode switching.

The robust control design methodology developed in [6], [7] makes use of certain assumptions about the magnitude of uncertain perturbations and interconnections between subsystems. Although perturbations and interconnection signals are not known, their magnitudes can be assumed to satisfy constraints expressed in terms of time domain IQCs of the form

$$
\begin{gathered}
\mathscr{E} \int_{0}^{t_{l}}\left(\left\|\zeta_{i}(t)\right\|^{2}-\left\|\xi_{i}(t)\right\|^{2}\right) d t \geq-x_{i 0}^{\prime} M_{i} x_{i 0} \\
\mathscr{E} \int_{0}^{t_{l}}\left(\sum_{\mu \neq i}\left\|\zeta_{\mu}(t)\right\|^{2}-\left\|r_{i}(t)\right\|^{2}\right) d t \geq-x_{i 0}^{\prime} \hat{M}_{i} x_{i 0} \\
\forall i=1, \ldots, N
\end{gathered}
$$

here $M_{i}=M_{i}^{\prime}>0, \hat{M}_{i}=\hat{M}_{i}^{\prime}>0$ and $\left\{t_{l}\right\}_{l=1}^{\infty}, t_{l} \rightarrow+\infty$, is a sequence of time instants; $\mathscr{E}$ denotes the expectation with respect to the underlying probability measure. The sets of admissible uncertainty inputs and admissible interconnection inputs $\xi_{i}(t), r_{i}(t)$, satisfying (8) and (9), will be denoted by $\Xi, \Pi$ respectively.

For the uncertain large scale system (7), (8), (9), we consider a decentralized output feedback absolute stabilization problem. The controllers considered are decentralized linear output feedback controllers of the form

$$
\begin{aligned}
& \dot{x}_{c, i}(t)=A_{c, i}(\eta(t)) x_{c, i}(t)+B_{c, i}(\eta(t)) y_{i}(t), \\
& u_{i}(t)=K_{c, i}(\eta(t)) x_{c, i}(t)
\end{aligned}
$$

where $x_{c, i} \in \mathbf{R}^{n_{c, i}}$ is the $i$ th controller state vector. The next section describes the underlying principles of the proposed technique and provides the control design algorithm.

\section{The Design Technique: Fundamentals And} ALGORITHM

Let $\tau_{i}>0, \theta_{i}>0, i=1, \cdots, N$, be given constants, and $\bar{\theta}_{i}=\sum_{j=1, j \neq i}^{N} \theta_{j}$. We consider a collection of the coupled generalized algebraic Riccati equations (GAREs) and generalized algebraic Riccati inequalities (GARIs):

$$
\begin{aligned}
& A_{i}^{\prime}(j) X_{i}(j)+X_{i}(j) A_{i}(j)+\bar{C}_{i}^{\prime}(j) \bar{C}_{i}(j)+\sum_{v=1}^{k} q_{j v} X_{i}(v) \\
& -X_{i}(j)\left[B_{i}(j) R_{i}^{-1}(j) B_{i}^{\prime}(j)-\bar{B}_{2, i}(j) \bar{B}_{2, i}^{\prime}(j)\right] X_{i}(j)=0, \quad(11) \\
& A_{i}^{\prime}(j) Y_{i}(j)+Y_{i}(j) A_{i}(j)+Y_{i}(j) \bar{B}_{2, i}(j) \bar{B}_{2, i}^{\prime}(j) Y_{i}(j) \\
& -\left[C_{y, i}^{\prime}(j) W_{i}^{-1}(j) C_{y, i}(j)-\bar{C}_{i}^{\prime}(j) \bar{C}_{i}(j)\right]+\sum_{v=1}^{k} q_{j v} Y_{i}(v)<0, \\
& j=1, \cdots, k,
\end{aligned}
$$

where $R_{i}(j)=\bar{D}_{i}^{\prime}(j) \bar{D}_{i}(j), W_{i}(j)=\bar{D}_{y, i}(j) \bar{D}_{y, i}^{\prime}(j)$ and

$$
\begin{aligned}
& \bar{C}_{i}(j)=\left[\begin{array}{c}
C_{i}(j) \\
\left(\tau_{i}+\bar{\theta}_{i}\right)^{1 / 2} H_{i}(j)
\end{array}\right], \quad \bar{D}_{i}(j)=\left[\begin{array}{c}
D_{i}(j) \\
\left(\tau_{i}+\bar{\theta}_{i}\right)^{1 / 2} G_{i}(j)
\end{array}\right], \\
& \bar{B}_{2, i}(j)=\left[\tau_{i}^{-1 / 2} E_{i}(j), \quad \theta_{i}^{-1 / 2} L_{i}(j)\right], \quad \bar{D}_{y, i}(j)=\left[\begin{array}{ll}
\tau_{i}^{-1 / 2} D_{y, i}(j), & 0
\end{array}\right] .
\end{aligned}
$$

Let $X_{i}(\eta(t)), Y_{i}(\eta(t))$ be defined as:

$$
X_{i}(\eta(t)):=X_{i}(j), \quad Y_{i}(\eta(t)):=Y_{i}(j), \quad \text { if } \eta(t)=j
$$

whenever $X_{i}(j), Y_{i}(j), j=1, \cdots, k$ exist.

Then associated with (11) and (12) is a collection of decentralized dynamic output feedback controllers of the form

$$
\begin{aligned}
\dot{x}_{c, i}(t)= & {\left[A_{i}(\eta(t))-\left(B_{i}(\eta(t)) R_{i}^{-1}(\eta(t)) B_{i}^{\prime}(\eta(t))\right.\right.} \\
& \left.\left.-\bar{B}_{2, i}(\eta(t)) \bar{B}_{2, i}^{\prime}(\eta(t))\right) X_{i}(\eta(t))\right] x_{c, i}(t) \\
& +\left[Y_{i}(\eta(t))-X_{i}(\eta(t))\right]^{-1} C_{y, i}^{\prime}(\eta(t)) W_{i}^{-1}(\eta(t)) \\
& \cdot\left[y_{i}(t)-C_{y, i}(\eta(t)) x_{c, i}(t)\right] \\
u_{i}(t)= & -R_{i}^{-1}(\eta(t)) B_{i}^{\prime}(\eta(t)) X_{i}(\eta(t)) x_{c, i}(t)
\end{aligned}
$$

Consider the following set of vectors:

$$
\begin{aligned}
& \mathscr{T}=\{\left\{\tau_{i} \quad \theta_{i}\right\}_{i=1}^{N} \in \mathbf{R}^{2 N}, \tau_{i}>0, \theta_{i}>0: \text { the set of } \\
& \text { coupled GAREs }(11) \text { admits a set of minimal } \\
& \text { positive definite solutions } X_{i}(j)>0, j \in \mathbb{K}, \text { and } \\
& \text { the set of coupled GARIs }(12) \text { admits a set of } \\
& \text { solutions } Y_{i}(j)>0, j \in \mathbb{K}, \text { such that } \\
&\left.Y_{i}(j)>X_{i}(j), \forall j \in \mathbb{K}\right\},
\end{aligned}
$$

According to [7], the sufficient and necessary condition for robust (absolute) stabilizability of the system (7) via the decentralized dynamic output feedback control (10) is that the set $\mathscr{T}$ is non-empty. Furthermore, when the set $\mathscr{T}$ is 
not empty, the worst case performance achievable using the decentralized controller (14) is bounded as follows:

$$
\begin{aligned}
& \inf _{\mathscr{U}} \sup _{\Xi, \Pi} \mathscr{E} \int_{0}^{+\infty} \sum_{i=1}^{N}\left\|z_{i}\right\|^{2} d t \\
& \leq \inf _{\mathscr{T}} \sum_{i=1}^{N} x_{i 0}^{\prime}\left[\sum_{j=1}^{k} \pi_{j} X_{i}(j)+\tau_{i} M_{i}+\theta_{i} \hat{M}_{i}\right] x_{i 0},
\end{aligned}
$$

where $\mathscr{U}$ denotes the set of all decentralized controllers (10). Suppose the infimum on the right-hand side of (15) is attained at $\tau_{i}^{*}, \theta_{i}^{*}, i=1, \cdots, N$. Then a decentralized controller satisfying this upper bound is given by (14) with the initial condition $x_{c, i}(0)=x_{i}(0)$ in which $\tau_{i}=\tau_{i}^{*}, \theta_{i}=\theta_{i}^{*}, i=$ $1, \cdots, N$.

In [8], the optimization problem on the right-hand-side of (15) was rewritten as a rank-constrained LMI problem. This rank-constrained LMI formulation is also given here in the Appendix. Although LMI formulations with rank constraints are typically non-convex problems (and therefore there is no available algorithm with a guaranteed proof of convergence to solve them), the LMIRank algorithm [12] was applied to solve this proposed optimization problem with good results. LMIRank uses SeDuMi [13] as its standard optimization tool, and can be called via YALMIP [14].

Now, based on the fundamentals described in this section, an algorithmic description of the proposed design procedure can be set up as follows:

Step 1: Build the nonlinear representation of the power system for each of the operating conditions (modes) of interest;

Step 2: Linearize each of the nonlinear sets of equations obtained in the previous step to build local models corresponding to each mode;

Step 3: With the linear models built in step 2, formulate the optimization problem (21) (as shown in the Appendix) and solve it (by e.g. the LMIRank solver) to obtain $\tau_{i}^{*}, \theta_{i}^{*}$ and $Y_{i}(j)$, for $i=1, \cdots, N$;

Step 4: Substitute the resulting $\tau_{i}^{*}$ and $\theta_{i}^{*}$ into GAREs (11) and solve (11) (either by an LMI approach [15] or an iterative approach [16]) to obtain $X_{i}(j)$;

Step 5: With the values obtained in the last two steps, calculate the controller description by the parameterization given in (14).

\section{EXAMPLE OF APPLICATION}

This section describes a successful application example of the proposed technique to the problem of oscillation damping in power systems. The events that produce the mode switching characteristics in the power system operation, in this case, are the tripping of a transmission line and its return to normal operation.

The power system model chosen for this application is a well-known benchmark system, extensively used in the study of electromechanical oscillations. Fig. 1 shows the oneline diagram of this system, and complete data for it can be obtained from [11].
In this work, instead of the usual double-circuit tie-line, a transmission line composed of 4 circuits was used to connect buses 7 and 9. However, the total impedance of the original tie-line was preserved in this modified model. This modification was necessary to avoid transient stability problems when one of the tie-line circuits trips. Another modification on the original data was the schedule of new generation settings as follows: $P_{G 1}=P_{G 2}=600 \mathrm{MW}$ and $P_{G 4}=800$ MW. Generator 3 was considered as an infinite bus, therefore being responsible for the adjustment of power imbalances and supplying an angular reference for the system.

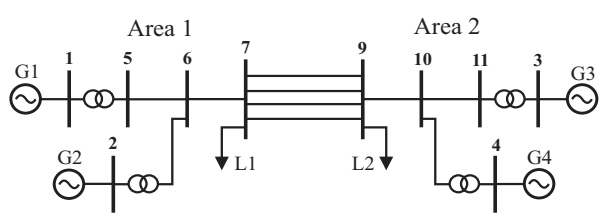

Fig. 1. Diagram of the benchmark example system.

For the system of Fig. 1, the 4-circuit tie-line exerts a great influence over the system modes of oscillation. Therefore, tripping of one of its circuits was considered in this paper as the event against which the designed controllers must provide robust stability and performance. In this case, the system can operate in two distinct modes. MODE 1 refers to its normal operating condition, while MODE 2 describes the operating point where only 3 of the tie-line circuits are connected.

Fig. 2 presents the eigenvalues (related to the electromechanical modes) of the open loop system for both modes. The poles related to MODE 1 are plotted as X-marks and the ones associated with MODE 2 as asterisks.

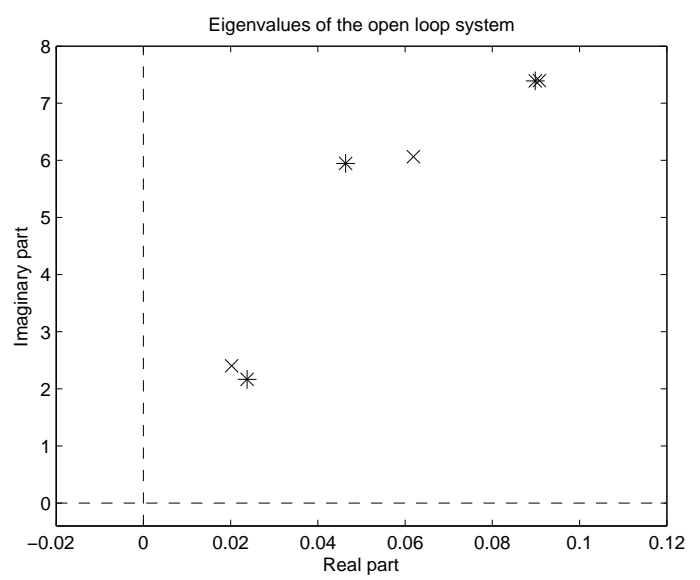

Fig. 2. Eigenvalues of the open loop system in both operating conditions (x - MODE 1; * MODE 2).

One can see that both open loop systems are unstable. So, an adequate design of damping controllers is essential for the system operation. Moreover, the switching between the two modes must be taken into account, so the system does not go unstable when the line trips. The technique presented in this paper was applied to address these requirements, and the results of this application were very satisfactory, as will be seen in the sequel. 
The ideal control variable to damp oscillations in power systems would be the mechanical power input of the generator. Unfortunately, this input is driven by the turbinegovernor loop, which is not fast enough to provide the required control action. The alternative [1] is the use of the AVR reference as the control input. However, since this alternative input has a limited effect over the dynamics of interest, the result is a very ill-conditioned problem.

Due to this ill-conditioning, SeDuMi reported some numerical problems when the LMIRank algorithm was called in Step 3. To overcome this difficulty, the $\theta_{i}$ and $\tau_{i}$ values at which SeDuMi terminated were fed into the nonlinear optimization function fmincon (available in the MATLAB Optimization Toolbox) and the upper bound on the infimum on the right hand side of (15) was calculated. In this process, the coupled GAREs (11) were solved using the algorithm outlined in [16] and GARIs (12) along with $Y_{i}(j)>X_{i}(j)$ were converted to their equivalent LMIs ${ }^{1}$.

In this paper, local uncertainties due to linearization errors were ignored, so we chose not to define local uncertainty outputs. Rather, we imposed a uniform upper bound on the energy of the interconnections. The IQC model (8), (9) provides for this, but also allows for other design approaches accounting for local uncertainties. The whole design process, as described in Section III, took approximately 10 minutes in a computer with a P4 $1.5 \mathrm{G}$ processor and $768 \mathrm{MB}$ of RAM memory. After that, the closed loop eigenvalues in both modes were checked to ensure the system is locally stable on both operating conditions. Also, the theory guarantees that the closed loop system is mean square stable. This provides a guarantee that switching between controllers will not lead to instability (in the mean-square sense). The minimum damping ratio of all closed loop system eigenvalues was $20.1 \%$ (as shown in Fig. 3). This is a very satisfactory result, given that a minimum damping of 5\% is usually accepted as a safe small-signal stability margin by the power industry.

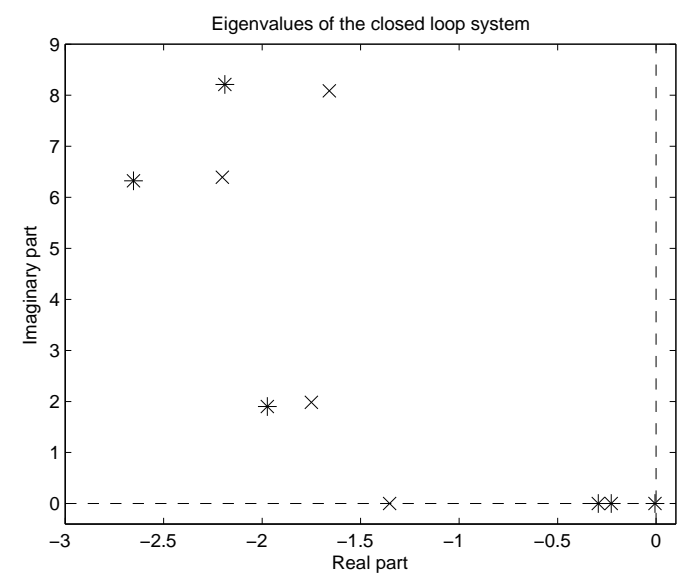

Fig. 3. Eigenvalues of the closed loop system in both operating conditions (x - MODE 1; * - MODE 2).

\footnotetext{
${ }^{1}$ For well-conditioned problems, the algorithm provided in the last section can be employed directly, without modification; this was confirmed by other examples, see e.g. [8].
}

However, the ultimate test for the designed controllers was carried out via nonlinear simulations of the tripping sequence. The first simulation was done under the assumption that the line trips, and no action is taken to reconnect it, so the system goes from MODE 1 to MODE 2 and then achieves a new equilibrium condition. For this simulation, Fig. 4 shows the rotor speed response of generators 1, 2 and 4. It can be seen that the controllers provide a stable and fast recover of the system after the occurrence of the line tripping (at $t=5 \mathrm{~s}$ ).

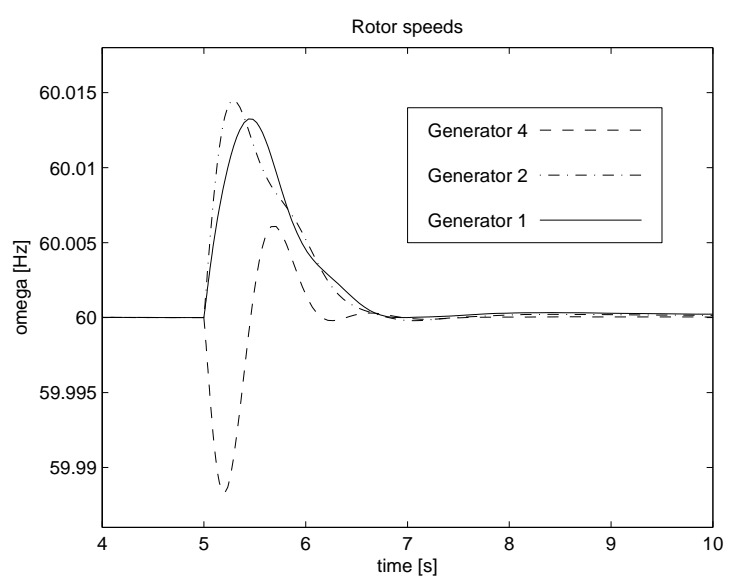

Fig. 4. Rotor speeds of generators respond to line tripping.

Furthermore, there are situations (mainly involving important transmission lines for the system), where some mechanism of automatic reconnection of the line is available. In these cases, the system may return to its original configuration in the middle of the transient initiated by the line tripping event. This could be a threat to the ability of some controllers to provide a stable response. On the other hand, the controllers designed by the technique proposed in this paper perform very well under these circumstances, as shown in Fig. 5 (where the line recloses at $t=5.5 \mathrm{~s}$ ).

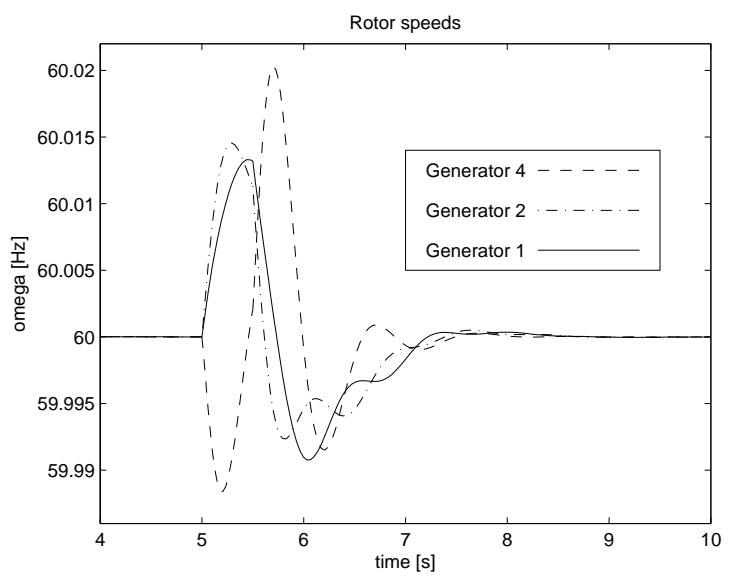

Fig. 5. Rotor speed response of generators (line trips and recloses). 


\section{CONCLUSIONS}

This paper presented the application of a new technique (based on the minimax optimal control theory of robust control of Markov Jump Parameter Systems) to the design of controllers to damp oscillations in power systems. The control design technique explicitly takes into account statistical information about the operating conditions of the system, which are typically available in power companies. By doing so, this technique can provide less conservative controllers, when compared to other control design approaches.

The presented results show that the designed controllers are able to properly stabilize the system, meeting the typically acceptable performance criterion. Moreover, they can withstand abrupt changes in the system, still providing a satisfactory response.

It is important to remark that this technique relies on some statistical knowledge about the power system, for the design stage, and an adequate on-line sensing scheme to detect the event occurrence, for the implementation stage. Therefore, it must be applied when these two requirements are available (such as problems where the oscillations are mainly related to tripping of specific lines or switching of particular loads, which can be predicted with enough accuracy and detected reasonably fast).

Further perspectives of this work include the application of this technique to systems with switching loads (with a statistically predictable behavior) and the design of supplementary controllers for FACTS devices.

\section{APPENDIX}

Consider the following rank constrained LMIs in the variables $W_{i}, \tilde{X}_{i}(j), F_{i}(j), Y_{i}(j), \tilde{\tau}_{i}, \tilde{\theta}_{i}$, and $\tau_{i}, \theta_{i}$ :

$$
\begin{aligned}
& {\left[\begin{array}{cccc}
\mathbf{N}_{i}(j) & \mathbf{V}_{i}(j) & F_{i}^{\prime}(j) D_{i}^{\prime}(j)+\tilde{X}_{i}(j) C_{i}^{\prime}(j) & \mathbf{Q}_{i}(j) \\
\star & -\mathbf{S}_{i}(j) & \mathbf{0} & \mathbf{0} \\
\star & \star & -\mathbf{I} & \mathbf{0} \\
\star & \star & \star & -\Theta_{i}
\end{array}\right]<0,} \\
& {\left[\begin{array}{ccc}
\mathbf{M}_{i}(j) & Y_{i}(j) E_{i}(j) & Y_{i}(j) L_{i}(j) \\
\star & -\tau_{i} \mathbf{I} & \mathbf{0} \\
\star & \star & -\theta_{i} \mathbf{I}
\end{array}\right]<0,} \\
& {\left[\begin{array}{cc}
\tilde{X}_{i}(j) & I \\
I & Y_{i}(j)
\end{array}\right]>0} \\
& {\left[\begin{array}{cccc}
W_{i} & \Pi_{i} & x_{i 0}^{\prime} M_{i}^{1 / 2} & x_{i 0}^{\prime} \hat{M}_{i}^{1 / 2} \\
\star & \tilde{\mathbf{X}}_{i} & \mathbf{0} & \mathbf{0} \\
\star & \star & \tilde{\tau}_{i} \mathbf{I} & \mathbf{0} \\
\star & \star & \star & \tilde{\theta}_{i} \mathbf{I}
\end{array}\right]>0} \\
& {\left[\begin{array}{cc}
\tilde{\tau}_{i} & 1 \\
1 & \tau_{i}
\end{array}\right] \geq 0, \quad \operatorname{rank}\left[\begin{array}{cc}
\tilde{\tau}_{i} & 1 \\
1 & \tau_{i}
\end{array}\right] \leq 1,} \\
& {\left[\begin{array}{cc}
\tilde{\theta}_{i} & 1 \\
1 & \theta_{i}
\end{array}\right] \geq 0, \quad \operatorname{rank}\left[\begin{array}{cc}
\tilde{\theta}_{i} & 1 \\
1 & \theta_{i}
\end{array}\right] \leq 1,} \\
& i=1, \cdots, N \text {, }
\end{aligned}
$$

where

$$
\begin{aligned}
\mathbf{N}_{i}(j)= & \tilde{X}_{i}(j) A_{i}^{\prime}(j)+A_{i}(j) \tilde{X}_{i}(j)+q_{j j} \tilde{X}_{i}(j)+B_{i}(j) F_{i}(j) \\
& +F_{i}^{\prime}(j) B_{i}^{\prime}(j)+\tilde{\tau}_{i} E_{i}(j) E_{i}^{\prime}(j)+\tilde{\theta}_{i} L_{i}(j) L_{i}^{\prime}(j), \\
\mathbf{V}_{i}(j)= & {\left[\sqrt{q_{j 1}} \tilde{X}_{i}(j) \cdots \sqrt{q_{j(j-1)}} \tilde{X}_{i}(j),\right.} \\
& \left.\sqrt{q_{j(j+1)}} \tilde{X}_{i}(j) \cdots \sqrt{q_{j k}} \tilde{X}_{i}(j)\right], \\
\mathbf{S}_{i}(j)= & \operatorname{diag}\left[\tilde{X}_{i}(1) \cdots \tilde{X}_{i}(j-1), \tilde{X}_{i}(j+1) \cdots \tilde{X}_{i}(k)\right], \\
\mathbf{Q}_{i}(j)= & {\left[F_{i}^{\prime}(j) G_{i}^{\prime}(j)+\tilde{X}_{i}(j) H_{i}^{\prime}(j)\right] \cdot[\mathbf{I}, \cdots, \mathbf{I}] \quad(N \text { entries }), } \\
\Theta_{i}= & \operatorname{diag}\left[\tilde{\tau}_{i} \mathbf{I}, \tilde{\theta}_{1} \mathbf{I}, \cdots, \tilde{\theta}_{i-1} \mathbf{I}, \tilde{\theta}_{i+1} \mathbf{I}, \cdots, \tilde{\theta}_{N} \mathbf{I}\right], \\
\mathbf{M}_{i}(j)= & A_{i}^{\prime}(j) Y_{i}(j)+Y_{i}(j) A_{i}(j)+\sum_{v=1}^{k} q_{j v} Y_{i}(v) \\
& -\tau_{i} C_{y, i}^{\prime}(j)\left[D_{y, i}(j) D_{y, i}^{\prime}(j)\right]^{-1} C_{y, i}(j) \\
& +C_{i}^{\prime}(j) C_{i}(j)+\left(\tau_{i}+\bar{\theta}_{i}\right) H_{i}^{\prime}(j) H_{i}(j), \\
\Pi_{i}= & {\left[\pi_{1}^{1 / 2} x_{i 0}^{\prime}, \cdots, \pi_{k}^{1 / 2} x_{i 0}^{\prime}\right], \tilde{\mathbf{X}}_{i}=\operatorname{diag}\left[\tilde{X}_{i}(1), \cdots, \tilde{X}_{i}(k)\right] . }
\end{aligned}
$$

The optimization problem on the right-hand-side of (15) is equivalent [8] to the following optimization problem:

$$
\begin{gathered}
\inf \left(W_{1}+\cdots+W_{N}\right) \quad \text { subject to (16-20). } \\
\text { REFERENCES }
\end{gathered}
$$

\section{REFERENCES}

[1] E. V. Larsen, and D. A. Swann, Applying Power System Stabilizers, Parts I, II and III, IEEE Trans. Power App. Syst., vol. PAS-100(6), 1981, pp. 3017-3043.

[2] B. Pal, and B. Chaudhuri, Robust Control in Power Systems, Springer, London, 2005.

[3] R. A. Ramos, L. F. C. Alberto, and N. G. Bretas, A New Methodology for the Coordinated Design of Robust Decentralized Power System Damping Controllers, IEEE Trans. Power Syst., vol. 19(1), 2004, pp. 444-454.

[4] W. Qiu, V. Vittal, and M. Khammash, Decentralized Power System Stabilizer Design Using Linear Parameter Varying Approach, IEEE Trans. Power Syst., vol. 19(4), 2004, pp. 1951-1960.

[5] R. A. Ramos, A. C. P. Martins and N. G. Bretas, An Improved Methodology for the Design of Power System Damping Controllers, IEEE Trans. Power Syst., vol. 20(4), 2005, pp. 1938-1945.

[6] V. A. Ugrinovskii, and H. R. Pota, Decentralized Control of Power System via Robust Control of Uncertain Markov Jump Parameter Systems, Int. J. Control, vol. 78(9), 2005, pp. 662-677.

[7] L. Li, and V. A. Ugrinovskii, Decentralized Robust Control of Uncertain Markov Jump Parameter Systems via Output Feedback, In: Proc. 2006 ACC, Minneapolis, USA, 2006, pp. 1340-5.

[8] L. Li, V. A. Ugrinovskii, and R. Orsi, Decentralized Robust Control of Uncertain Markov Jump Parameter Systems via Output Feedback, submitted to Automatica.

[9] I. R. Petersen, V. A. Ugrinovskii, and A. V. Savkin, Robust Control Design Using $H_{\infty}$ Methods, Springer-Verlag, London, 2000.

[10] P. M. Anderson, and A. A. Fouad, Power System Control and Stability, IEEE Press, Piscataway, 1994.

[11] P. Kundur, Power System Stability and Control, McGraw-Hill, New York, 1994.

[12] R. Orsi, LMIRank: Software for Rank Constrained LMI Problems, Available from http://rsise.anu.edu.au/ robert/lmirank/.

[13] J. F. Sturm, Using SeDuMi 1.02, a Matlab Toolbox for Optimization over Symmetric Cones (Updated for Version 1.05), 2001. Available from http://sedumi.mcmaster.ca/.

[14] J. Löfberg, "YALMIP: A Toolbox for Modeling and Optimization in MATLAB", In: Proc. CACSD Conf., Taipei, Taiwan, 2004. Available from http://control.ee.ethz.ch/ joloef/yalmip.php

[15] M. Ait Rami, and L. El Ghaoui, LMI Optimization for Nonstandard Riccati Equations Arising in Stochastic Control. IEEE Trans. Automatic Control, vol. 41, no. 11, 1996, pp. 1666-1671.

[16] Z. Pan, and T. Basar, $H_{\infty}$ Control of Markovian Jump Systems and Solutions to Associated Piecewise-Deterministic Differential Games, In: New Trends in Dynamic Games and Applications (G. J. Olsder, ed.), Birkhäuser, Boston, 1995, pp. 61-94. 